\title{
Motivational Pathways to Social and Pedagogical Inclusion in Physical Education
}

\section{Terese Wilhelmsen, Marit Sørensen, and Ørnulf N. Seippel Norwegian School of Sport Sciences}

\begin{abstract}
This article is focused on how combinations of motivational attributes and motivational climates support social and pedagogical inclusion in physical education among children with disabilities. Theoretically, the authors integrate tenets from achievement-goal theory and self-determination theory. To capture the motivational complexity underlying children's experiences of inclusion in physical education, they use a 2-step fuzzy qualitative comparative analysis. The analyses of contextual conditions yielded 2 sufficient inclusion-supportive climates, namely a physically inclusive and mastery-oriented climate or a physical inclusive, autonomy-supportive, and low performance-oriented climate. The configurations of motivational attributes in the inclusion-supportive climates indicated 4 sufficient pathways to social and pedagogical inclusion. The path with the largest coverage of children was in the physically inclusive and masteryoriented climate and represented children who were task and ego oriented and low on amotivation and experienced satisfaction of the need for autonomy, competence, and relatedness.
\end{abstract}

Keywords: achievement-goal theory, children with disabilities, inclusive education, QCA, self-determination theory

What does it take to support inclusion in physical education (PE)? This is an important question given the globalization of the inclusive PE ideology, yet it has received scant attention in previous literature (Wilhelmsen \& Sørensen, 2017, with the exceptions of Dunn \& Dunn, 2006; Obrusnikova \& Dillon, 2012; Pan, Tsai, Chu, \& Hsieh, 2011; Valentini \& Rudisill, 2004). In this study, we employ tenets from achievement goal theory (AGT) and self-determination theory (SDT) to explore what motivational pathways support social inclusion (SI) and pedagogical inclusion (PI) in PE as perceived by children with disabilities.

Combining tenets from AGT and SDT allow us to explore relations between theoretically distinct aspects of the motivational processes that we posit are essential to understanding inclusion in PE. Despite differences in basic assumptions

The authors are with the Norwegian School of Sport Sciences, Oslo, Norway. Wilhelmsen (terese. wilhelmsen@nih.no) is corresponding author. 
of what drives human behavior in achievement contexts-for example, perceived motivational climate and goal orientations (AGT: Roberts, 2012) versus satisfaction of innate psychological needs (SDT: Deci \& Ryan, 2000), extensive research has shown the value of combining the two theories (Cox \& Williams, 2008; Duda, 2013; Ommundsen \& Kvalø, 2007; Spray, Wang, Biddle, \& Chatzisarantis, 2006; Standage, Duda, \& Ntoumanis, 2003).

A challenge of studying the motivational mechanisms involved in inclusion in $\mathrm{PE}$ is the potential complexity of associations: many factors are involved, they can be combined in different ways, and several pathways to the same outcome may exist. To be able to capture this complexity, we use a two-step fuzzy qualitative comparative analysis (QCA; see "Method" section). Using QCA allowed us to uncover motivational pathways linked to both contextual conditions (i.e., motivational climates and degree of physical inclusion) and individual attributes (i.e., psychological needs satisfaction, motivation orientation, and motivation regulation) that support inclusion in PE. QCA is well suited for grasping complex and asymmetric relations compared with traditional statistical inference, which has been commonly used in the literature (Braithwaite, Spray, \& Warburton, 2011; Ragin, 2008; Van den Berghe, Vansteenkiste, Cardon, Kirk, \& Haerens, 2014).

The question we wish to answer is "what motivational pathways support social and pedagogical inclusion in PE among children with disabilities in PE?" From a theoretical standpoint, the integration of AGT and SDT tenets using a QCA approach is a useful contribution to our base of knowledge because it may lead to a new, and perhaps enriched, understanding of the diverse psychological processes involved in PE. From a practical standpoint, the enhanced knowledge may help us understand how teachers can facilitate a learning climate that promotes inclusion for all children.

In the following sections, we define and describe our understanding of inclusion in PE. Second, based on previous research, we show how AGT and SDT tenets promote a comprehensive understanding of the motivational processes involved inclusion in PE. Given the novelty of QCA in sport science research, we introduce the main concepts of QCA in the "Method" section. Results are presented in two steps. First, we focus on the contextual conditions that might support inclusion in PE before we introduce the individual motivational attributes in the second step. Finally, we sum up and discuss our findings, possible implications, and strengths and weaknesses of the study.

\section{Inclusion in PE}

We understand inclusion as a multidimensional phenomenon and distinguish between SI, PI, and physical inclusion (Dalen, 1994). SI refers to the interaction between children and their peers, between children and the teachers in class, as well as the children's experiences of belonging to the group. Studies on inclusion in PE have consistently shown the importance of a learning environment that promotes positive interaction, and children with disabilities have stressed the importance of having friends, feeling supported, and being a legitimate participant in physical activities (Klavina \& Block, 2008; Seymour, Reid, \& Bloom, 2009; SpencerCavaliere \& Watkinson, 2010). 
The pedagogical dimension of inclusion reflects the degree to which the organization of PE allows all children to use their abilities, and promotes children's learning potential and engagement in the activities. Finally, physical inclusion relates to the degree to which children with disabilities participate in PE with peers. Contemporary research consistently acknowledges that inclusion is more than mere placement. However, we postulate that SI and PI depends on the degree of physical inclusion. The three dimensions are not discrete entities, but interreliant dimensions. They may interact differently dependent on the interaction between individual and contextual motivational conditions.

\section{Contextual Conditions: Perceived Motivational Climates in PE}

Three motivation climates (i.e., mastery, performance, and autonomy-supportive climates) serve as the contextual conditions in the analysis in addition to physical inclusion. The distinction between mastery and performance climates is from the sociocognitive AGT framework (Roberts, 2012). In a mastery climate, the PE teacher emphasizes mastery of task and effort as a criteria of success, mistakes as an essential part of learning, and self-referenced learning (Braithwaite et al., 2011). One assumption in AGT is that teachers' promotion of effort and improvement supports children's perceptions of ability as a dynamic entity that improves with effort. In contrast, a performance climate fosters normative understandings of ability and other-referenced criteria for success and failure (Roberts, 2012). A PE teacher promotes a performance climate by underlining social comparison and interpersonal competition, by punishing mistakes, and by providing differentiated feedback based on a normative understanding of ability. A performance climate is believed to create PE contexts that celebrate mastery experiences for the few, specifically for the children who are able to outperform their peers. Because experiences of success would depend on continuous outperformance, a performance climate creates a vulnerable situation even for the children with high normative ability. The focus on normative ability in PE may also impede positive peer relations and lead to rivalry and devaluation of diversity (Obrusnikova \& Dillon, 2012). Previous studies support the association between perceived mastery climate and positive correlates such as enjoyment, task orientation, and confidence in sport and PE, and the link between a performance climate and negative correlates such as anxiety, boredom, and ego orientations (Ntoumanis \& Biddle, 1999). Furthermore, a meta-analysis of motivational interventions in PE indicated small positive effects for participants in mastery climate contexts, with the largest effects being behavioral outcomes (i.e., skill development, health, and fitness), followed by affective (i.e., enjoyment) and cognitive (i.e., confidence, learning strategies, perceived climate, and task orientation) outcomes (Braithwaite et al., 2011).

Self-determination theory is based on the assumption that innate psychological needs are the basis of our self-motivation and optimal functioning and that the satisfaction or thwarting of these needs is contextually conditioned (Deci \& Ryan, 2000). Autonomy support from teachers is believed to positively influence basic needs satisfaction and subsequent autonomous motivation (Edmunds, Ntoumanis, \& Duda, 2007; Mageau \& Vallerand, 2003). To promote an autonomy-supportive 
climate, the PE teacher should be engaged, respectful of children's perspectives, provide information-rich feedback on children's competence, and promote choice and initiative within a structured learning environment (Edmunds et al., 2007; Hastie, Rudisill, \& Wadsworth, 2013). In comparison, controlling environments in which behavior is controlled by self-imposed pressure, feedback based on normative evaluation, external rewards, or punishment is likely to generate perceptions of incompetence and undermine children's intrinsic motivation (Hein \& Koka, 2007). In this climate, engagement in tasks or a specific behavior is dependent on the contingency of the extrinsic reinforcement.

Previous research has found several links between tenets from AGT and SDT. A common link is between mastery climate and more self-determined motivation and performance climate and less self-determined motivation (Ommundsen \& Kvalø, 2007; Parish \& Treasure, 2003; Standage et al., 2003). For example, a study found perceived mastery climate and autonomy support to positively influence intrinsic motivation and negatively influence amotivation in $\mathrm{PE}$, and perceptions of performance climate were positively associated with amotivation (Ommundsen \& Kvalø, 2007). Furthermore, an experimental study found that children in autonomy-supportive environment, regardless of their achievement involvement, experienced greater enjoyment, performed better, and persisted longer at the task than children in the controlling communication condition (Spray et al., 2006). With this in mind, we expected that perceived mastery and autonomy-supportive climates would support perceptions of SI and PI in PE among children with disabilities, whereas a perceived performance climate would impede inclusion.

\section{Individual Attributes: Motivation Orientations, Basic Psychological Need Satisfaction, and Motivation Regulations}

The individual attributes believed to influence children's experiences of SI and PI are their motivation orientation (i.e., task and ego orientation) and their basic psychological need satisfaction (i.e., satisfaction of autonomy, relatedness, and competence, their motivational regulations, and amotivation for PE).

Within AGT, predispositions for task and ego involvement are referred as task and ego orientation (Nicholls, 1989). Goal orientations are posited as dynamic entities and children's experiences at home, through sport, or other arenas can influence their personal goal orientations in PE. Research has shown that the most adaptive motivational attributes are either high task and high ego orientation or high task and low ego orientation (Roberts, 2012). Perceived ability is believed to interact with task and ego goals. For a task-oriented child focused on selfreferenced learning, perception of ability may be less relevant for engagement in a task. In contrast, an ego-oriented child who perceives herself high on ability and likely to outperform others may be more prone to engage in a task where success is believed to be demonstrative of high normative ability. Whereas an ego-oriented child with low perceptions of ability may refrain from taking part, or self-handicap, if the perceived likelihood of outperforming others is low, or if demonstration of low normative performance is perceived as a failure (Nicholls, 1989; Parish \& Treasure, 2003). 
In SDT, how we interpret the perceived relation of situations to our basic psychological needs serve as important determinants of human behavior (Deci \& Ryan, 2000). The fundamental psychological needs are autonomy, relatedness, and competence. The need for autonomy concerns the feeling of being "the origin or source of one's own behavior" (Ryan \& Deci, 2002, p. 8). The need for relatedness concerns the feeling connected to and accepted by others, and the feeling of belonging within a group or community (Ryan \& Deci, 2002). The need for competence can be defined as "feeling effective in one's on-going interaction with the social environment and experiencing opportunities to exercise and express one's capacities" (Ryan \& Deci, 2002, p. 7). Satisfaction of the need for competence is believed to lead to individuals seeking challenges aligned with their capacities and to strive for enhancement of those skills. According to the subtheory of basic psychological needs, the extent of needs satisfaction dictates the level of selfdetermined motivation (Deci \& Ryan, 2000). Previous findings show that fulfilment of basic needs leads to enhanced enjoyment in PE (Ommundsen \& Kvalø, 2007).

Self-determination theory extends the traditional distinction between intrinsic and extrinsic motivation by proposing that a continuum is formed by intrinsic motivation and varied forms of extrinsic motivations based on the reasons that give rise to an action (Deci \& Ryan, 2000). In this study, the motivation regulation toward engaging in PE was measured with the relative autonomy index (RAI; Grolnick \& Ryan, 1987), which distinguishes between different qualities of motivation on a continuum from more controlled (external and introjected regulations) motivations toward more autonomous (identified and intrinsic regulations) motivations (Deci \& Ryan, 2000; Grolnick \& Ryan, 1987). In addition, amotivation measured the degree to which a child lacks motivation and intentions to act in PE.

Theorists from both camps (Deci \& Ryan, 2000; Duda, 2013; Nicholls, 1989) have proposed links between the two theories despite different basic assumptions. As previously indicated, both AGT and SDT emphasize the relations between perceived climate, individual motivational attributes, and positive or negative outcomes (Deci \& Ryan, 2000; Nicholls, 1989; Roberts, 2012). Previous findings indicate a positive relationship between task orientation and more self-determined motivation, and a positive relationship between ego orientation and less selfdetermined motivation (Parish \& Treasure, 2003). Moreover, a latent profile analysis of homogeneous attributes of perceived motivational (e.g., mastery, performance, autonomy supportive, and relatedness supportive) climates in PE identified five clusters that influenced enjoyment in PE differently (Jaakkola, Wang, Soini, \& Liukkonen, 2015). However, we need more knowledge on how various configurations of motivational climates and motivational attributes influence children's experiences with PE.

Figure 1 depicts a graphical summary of potential configurational relations between motivational climates, motivational attributes, and perceived SI and PI in PE. Figure 1 presents the interplay of motivational conditions that we expect support or hinder perceived inclusion in PE. With four contextual conditions and seven individual attributes, there are 142 possible pathways $\left(\left(2^{k}-1\right)+\left(2^{k}-1\right)=\right.$ $n)$, and the question is which of these are conducive to inclusion. QCA helps us study various pathways, and once the motivational paths are identified, it is possible to specify and explain the combination of contextual conditions and individual attributes that support SI and PI in PE. 


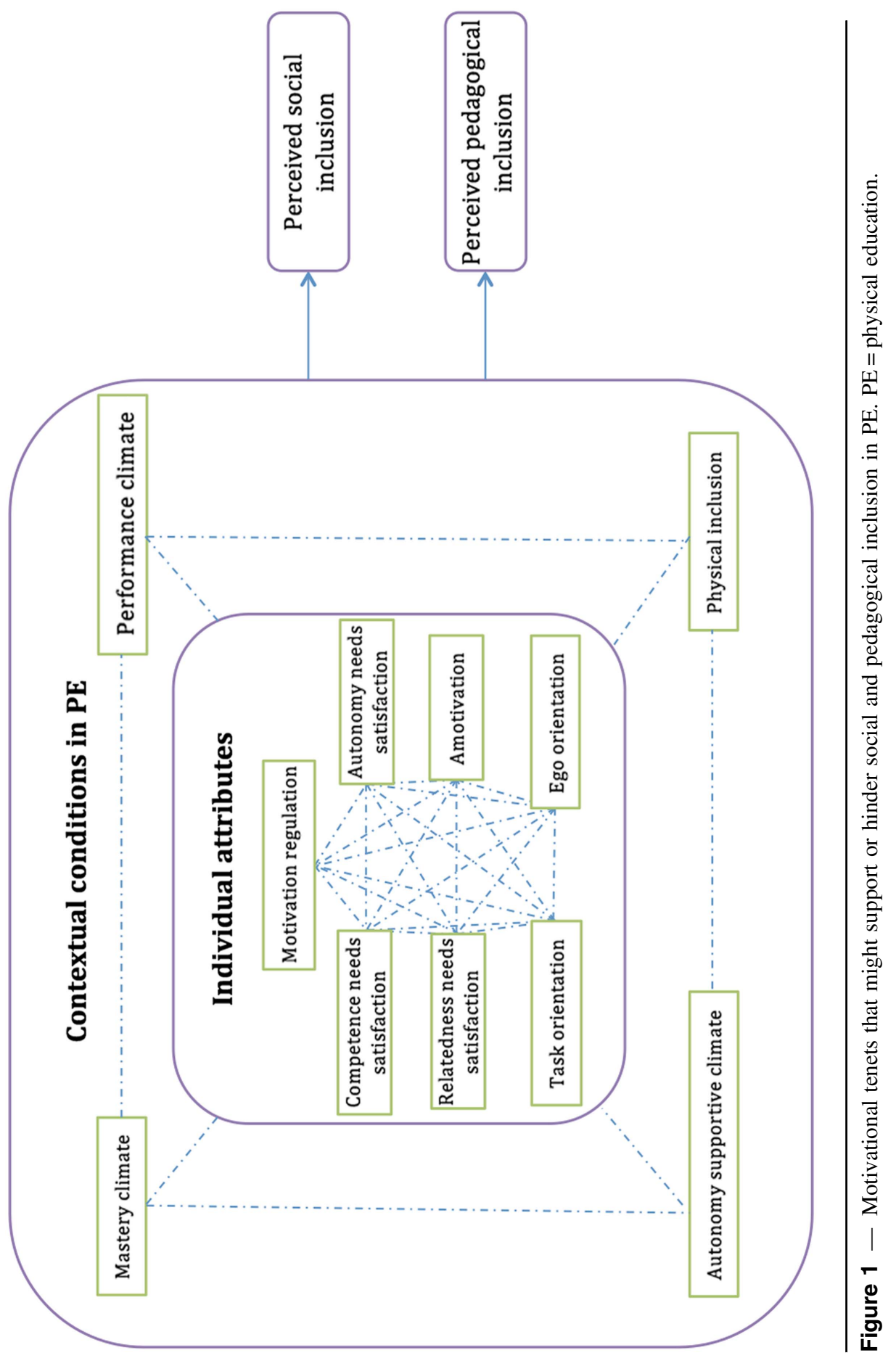


Based on the underlying principles of QCA, three ideas are central to our modeling: (a) alternative pathways to SI and PI are possible (i.e., equifinality); (b) one condition may not display an effect on children's perceptions of inclusion on its own, but only together with other conditions (i.e., conjunctional causation); and (c) both the presence and absence of a condition might lead to SI and PI, depending on the configuration (i.e., asymmetrical causation).

\section{Method}

\section{Participants and Procedures}

The participants were 64 children with disabilities ( 28 girls, 34 boys, and two did not report sex; 7-16 years of age, $M=13.23, S D=2.05$ ) attending Norwegian elementary schools (Grade 2-10). Among them 33\% had a physical disability, $28 \%$ had cerebral palsy, $8 \%$ had a developmental disability, $5 \%$ a visual disability, $5 \%$ a learning disability (including attention deficit hyperactivity disorder), and $3 \%$ had autism spectrum disorder. According to parental reports, $6 \%$ of the children had a high degree of disability, $28 \%$ had moderate disability, $42 \%$ had a mild degree of disability, and $6 \%$ reported having no disability. (Eleven parents did not specify their child's type or degree of disability.)

A cross-sectional design was used in this study. We initially attempted to contact children and their parents through a school-based national representative sample. However, the response rate and the diversity of disabilities among the children were low. (There was $9 \%$ response rate, and the majority of the children had asthma.) Thus, in our second attempt, we used a convenience sample approach. Children with disabilities and their parents were informed about the project through a letter in collaboration with regional rehabilitation centers or verbally informal parental meetings at one rehabilitation center specialized in adapted physical activity. Children were given the option of responding to an online or a hard copy version of the survey. For the online version, we used SurveyXact (Århus, Denmark) with which the Norwegian School of Sport Sciences had a data handling agreement. Parents were encouraged to assist their children if needed. The Norwegian Center for Research Data approved the study. Both parents and children signed an informed consent form with information about the project and their participatory rights.

\section{Measures}

Social Inclusion. To measure SI, we designed a 12-item with a 5-point Likert type scale inspired by the Norwegian version of the Booth Index of Inclusion (Booth \& Ainscow, 2002). We used Pearson correlation, principal component analysis, and oblimin with Kaiser normalization rotation to identify the factor structure of the items (Tabachnick \& Fidell, 2014). Items with correlation $r=.30$ or less on the marker item (e.g., "In PE, I feel like a part of the class") were excluded from the analysis (one item excluded). Using the pattern matrix, items loading .32 on two or more factors were excluded (one item excluded). Next, the principal component analysis indicated two factors without cross-loadings. (The first factor had eight items, and the second factor had two items.) The eight-item factor was averaged to construct one scale measuring SI (Kaiser-Meyer-Olkin $=0.87, \alpha=.87,56 \%$ of variance). 
Pedagogical Inclusion. A similar process was followed for the PI scale inspired by the Norwegian version of the Booth Index of Inclusion (Booth \& Ainscow, 2002). Five out of 12 items were deleted based on low correlations with the marker item (e.g., "I learn something every PE lesson"). The factors analysis of the remaining seven items indicated one factor (Kaiser-Meyer-Olkin $=0.77, \alpha=.78,46 \%$ of variance).

Motivational Climates. We used a short Norwegian version of the perceived motivational climate in sport questionnaire (Sørensen, Roberts, \& Farholm, 2018) to measure mastery climate and performance climate. Each subscale consisted of three items. Responses were made on a 5-point Likert type scale ranging from 5 (very true) to 1 (not at all true) preceded by the stem: "My PE teacher thinks I am successful when ... " An example item is: ". . . I learn new skills." Items from each subscale were averaged to construct a mastery climate scale $(\alpha=.82)$ and performance climate scale $(\alpha=.89)$.

Autonomy Support. To assess children's perception of an autonomy-supportive environment in PE, we used the learning climate questionnaire modified to PE (Standage, Duda, \& Ntoumanis, 2006). The children answered on a 5-point Likert type scale ranging from 5 (strongly agree) to 1 (strongly disagree) preceded by the stem "In the PE classes ..." An item example is ". . . we feel that the PE teacher provides us with choice and options." Items were averaged to construct one scale $(\alpha=.79)$.

Physical Inclusion. The item "how often do you take part in PE together with your peers" was used to measure physical inclusion. Responses were made on a 5-point Likert type scale from 1 (never) to 5 (always).

Motivational Orientations. We used a short Norwegian version of the perception of success questionnaire to measure motivational orientations (Sørensen et al., 2018). Each subscale consists of three items. Responses were made on a 5-point Likert type scale from 5 (very true) to 1 (not at all true), preceded by the stem: "In PE, I feel successful when . . ." An example item is: “. . . I am the best.” Items from each subscale were averaged to construct a task orientation scale $(\alpha=.82)$ and an ego orientation scale $(\alpha=.87)$.

Basic Psychological Needs Satisfaction. Need satisfaction of autonomy was assessed with a 5-item scale used in previous studies (Standage et al., 2003, 2006). Participants responded to the items (e.g., "In PE I have some choice over what I do") on a 5-point Likert type scale ranging from 5 (strongly agree) to 1 (strongly disagree). Items were averaged to construct one scale $(\alpha=.76)$. Need satisfaction of competence was assessed using the 5-item Perceived Competence subscale of the Intrinsic Motivation Inventory modified to the PE (Standage et al., 2003, 2006). Participants responded to the items (e.g., "I am satisfied with my performance in PE") on a 5-point Likert type scale ranging from 5 (strongly agree) to 1 (strongly disagree). Items were averaged to construct one scale $(\alpha=.76)$. Need satisfaction of relatedness was assessed using the acceptance subscale of the Need for Relatedness Scale modified to the PE setting by Standage et al. (2003, 2006). Preceded by the stem: "With the other pupils in the PE classes I feel . . .," participants responded to five items (e.g., "“. . supported”) on a 5-point Likert type 
scale ranging from 5 (strongly agree) to 1 (strongly disagree). Items were averaged to construct one scale $(\alpha=.93)$.

Motivational Regulations. Motivational regulations were assessed with the use of an instrument devised by Goudas, Biddle, and Fox (1994). The perceived locus of causality measures four subscales of motivational regulation: internal motivation $(\alpha=.92)$, identified regulation $(\alpha=.92)$, introjected regulation $(\alpha=.61)$, and external regulation $(\alpha=.75)$, whereas the amotivation subscale was based on the Academic Motivational Scale $(\alpha=.91)$. Each subscale consisted of four items on 5point Likert scale ranged from 5 (very true) to 1 (not at all true) preceded by the stem "I take part in PE class ..." Previous work has supported the psychometric properties of the instrument (Goudas et al., 1994; Standage et al., 2003). For parsimony, we computed an index of motivation regulation labeled RAI followed by the procedures suggested by Grolnick and Ryan (1987). The RAI form one continuous variable from less to more self-determined styles of motivation and studies have indicated that the RAI adequately assesses self-determination in school and sport (Grolnick \& Ryan, 1987; Ommundsen \& Kvalø, 2007). Amotivation was introduced as a separate condition in the analyses to measure lack of motivation for PE.

Disability. Parents were asked to describe the type and degree of their child's impairment. Based on our former experiences, we know that some parents try to limit the focus on disability labels by not discussing diagnostic issues with their child. Thus, due to ethical considerations, we did not ask children themselves to specify their type or degree of impairment. Degree of impairment was measured in terms of no disability, low, moderate, and severe disability.

\section{The QCA Approach}

Qualitative comparative analysis is a relatively new analytical approach proven valuable in educational research and beyond (Cooper \& Glaesser, 2011; Ragin, 2008; Thiem \& Dusa, 2013). The main aims of QCA are (a) to unravel causal complexity by examining cases that share combinations of conditions to see if they also share the same outcome and (b) to interpret relations between the conditions and the outcome in terms of necessity and/or sufficiency (Schneider \& Wagemann, 2006). Next, we introduce the basic concepts of QCA. We recommend Ragin (2008) for in-depth descriptions.

Necessity Relations. A condition is necessary if, whenever we see the outcome, then we also see the condition. An example is if every time children feel pedagogically included in PE they also report being physically included, then physical inclusion is a necessary condition for PI. However, it is possible for a child to be physically included without feeling pedagogically included. Another example is all pregnant people are women (necessarily), but not all women are pregnant.

Sufficiency Relations. A condition is sufficient if whenever we see the condition then we also see the outcome. An example of a sufficient configuration (i.e., when a combination of two or more conditions are sufficient, but not the individual 
conditions by themselves) is if every instance a child reports high perception of mastery climate and task orientation (MAS*TAS), she also reports high perception of SI. Then mastery climate combined with task orientation is a sufficient configuration for (SI $\leftarrow$ MAS*TAS). However, we could still observe a child who feels socially included in PE, but with low perception of mastery climate and task orientation.

Logic and Boolean Algebra. The underlying epistemology and mathematical models in QCA differ from traditional statistical inference, and the notations and tables require a different interpretation. QCA uses formal logic and Boolean algebra to express the relationships between conditions and the outcome. The three basic operators are: logical OR (+) represents the union of conditions in which either of the conditions would lead to the outcome; logical AND (*) represents the intersection of conditions wherein the outcome is dependent on the concurrence of the conditions; and logical NOT in which negations of a conditions are denoted by replacing uppercase letters with lowercase letters. If we take the example above and add a second path toward SI, an autonomysupportive climate AND negation of amotivation (AUS*amo), the formula would read SI $\leftarrow$ MAS*TAS + AUS*amo. The formula specifies two sufficient, yet distinct, paths toward SI, namely mastery-oriented climate for children who are task oriented OR an autonomy-supportive climate for children who are not amotivated. Whether we can interpret the formula as causal paths need to be theoretically determined (Ragin, 2008).

INUS Conditions. QCA enables the identification of conditions that are "insufficient but necessary part of a condition which itself is unnecessary but sufficient for the result (INUS)" (Mackie, 1965 as cited in Ragin, 2008, p. 154). In the fictitious formula above, all four conditions are INUS conditions. For example, take the MAS condition: MAS is an INUS condition because it does not yield the results on its own, but only in combination with the TAS condition. Furthermore, MAS*TAS is a sufficient path, but not necessary given the existence of the alternative path AUS*amo. INUS conditions are phenomena beyond the reach of conventional statistical analysis (Ragin, 2008).

Fuzzy QCA. While an earlier version of QCA (crisp QCA) required a binary classification of the conditions and outcomes, the more recent fuzzy QCA (fsQCA) allows for degrees of membership by assigning fuzzy membership scores. Fuzzy membership scores imply the degree to which different cases belong to a set/condition (including full membership, the point of crossover, and full nonmembership; Ragin, 2008). The calibration of thresholds is both a qualitative and a quantitative approach in that they are assigned on the basis of theoretical knowledge and empirical evidence (Ragin, 2008). Membership scores in the range $0.5-1$ represent cases that are more "in" than "out" of a given condition, whereas the opposite is true for scores in the range $0-0.5$. Score equal to 0.5 represents the point of maximum ambiguity and are thought of as neither "in" nor "out" of the condition. After the calibration of the variables, memberships in the different conditions are compared to identify necessity and sufficiency relations between the conditions and the outcome. 
Two-Step fSQCA. In an effort to reduce the complexity of the results and to reduce the challenge of limited diversity (i.e., logical possible configurations of conditions that do not appear empirically), we approach the fsQCA in two steps. In a two-step fsQCA, conditions are divided into two groups based on differences in proximity (Schneider \& Wagemann, 2006). In this study, the conditions are divided into contextual conditions (motivational climates and physical inclusion) and individual attributes (motivational orientation, motivation regulation, and satisfaction of basic psychological needs). The two groups of conditions are introduced in the analyses in two steps. In the first step, we analyze the relations between the contextual conditions and perceived inclusion to identify different combinations of inclusive-supportive contexts. In the second step, we explore the combinations of individual attributes within the inclusion-supportive climates that jointly lead to SI and PI in PE.

Measures of Fit. The measure of consistency (con) indicates the degree to which cases with the outcome also exhibit the conditions and corresponds to the role of the $p$ value in statistical inference. Perfect consistency would imply that all cases with the same pattern of conditions would exhibit the outcome. However, perfect consistency is rare (Ragin, 2008). fsQCA supports quasisufficient relations by allowing a small number of cases to deviate from the patterns elucidated in the analysis. As recommended by Ragin (2008), we allow limited inconsistency in the analysis with a minimum consistency score of 0.85 . Raw coverage (cov.r) measures the degree to which the conditions in the solution formula explain all cases with the outcome and resembles the $R^{2}$ measure in regression analysis. Unique coverage (cov.u) measures the partitioning coverage of each configuration in the formula. Finally, the proportional reduction in consistency $(P R I)$ measures the reduction inconsistency if one configuration is left out of the model.

\section{Data Analyses}

We used IBM SPSS Statistics 24 (IBM Corp., Armonk, NY) and R 3.4.1 (R Core Team, Vienna, Austria) as platforms for the analyses. To handle missing values in the data, we used the $R$ package "Multivariate Imputation by Chained Equations (MICE)" (Van Buuren \& Groothuis-Oudshoorn, 2011). To perform the two-step fsQCA analyses (Schneider \& Wagemann, 2006), we used the $R$ package "QCAQUI" (Dusa, 2007, 2013). We tested the models for parameter sensitivity and robustness with the use of the systematic procedures promoted by Skaaning (2011).

\section{Results}

\section{Descriptive Statistics}

Mean, minimum score, maximum score, and configuration thresholds of the outcome and antecedent conditions are shown in Table 1. The descriptive statistics give an overview of all variables introduced in the analyses before the calibration into set relations. Lower and upper thresholds of the conditions are based on the 0 and 100 percentiles. The crossover threshold is the 3 (middle score) in the 5-point 


\section{Table 1 Descriptive Statistics of the Outcome and Antecedent Conditions}

\begin{tabular}{llllcc}
\hline Conditions & Code & Mean & Minimum & Maximum & LT/CT/UT \\
\hline Outcome & & & & & \\
$\quad$ social inclusion & SI & 4.24 & 1.50 & 5.00 & $1.50 / 3.00 / 5.00$ \\
$\quad$ pedagogical inclusion & PI & 3.50 & 1.43 & 5.00 & $1.43 / 3.00 / 5.00$ \\
$\begin{array}{l}\text { Context } \\
\text { mastery climate }\end{array}$ & MAS & 3.84 & 1.00 & 5.00 & $1.00 / 3.00 / 5.00$ \\
performance climate & PER & 2.75 & 1.00 & 5.00 & $1.00 / 3.00 / 5.00$ \\
autonomy support & AUS & 3.59 & 1.00 & 5.00 & $1.00 / 3.00 / 5.00$ \\
physical inclusion & PHY & 4.63 & 1.00 & 5.00 & $1.00 / 3.00 / 5.00$ \\
Individual & & & & & \\
task orientation & TAS & 4.56 & 1.33 & 5.00 & $1.33 / 3.00 / 5.00$ \\
ego orientation & EGO & 3.43 & 1.00 & 5.00 & $1.00 / 3.00 / 5.00$ \\
need for autonomy & AUT & 3.83 & 1.00 & 5.00 & $1.00 / 3.00 / 5.00$ \\
need for competence & COM & 3.37 & 1.00 & 5.00 & $1.00 / 3.00 / 5.00$ \\
need for relatedness & REL & 4.13 & 1.20 & 5.00 & $1.20 / 3.00 / 5.00$ \\
relative autonomy index & RAI & 2.52 & -9.25 & 10.25 & $-9.00 / 0.00 / 9.00$ \\
amotivation & AMO & 1.91 & 1.00 & 5.00 & $1.00 / 3.00 / 5.00$ \\
\hline
\end{tabular}

Note. $\mathrm{LT}=$ lower threshold; $\mathrm{CT}=$ crossover threshold; UT $=$ upper threshold.

Likert scale, which represents maximum ambiguity (i.e., the crossover point from more in than out of the set). One exception is the RAI (ranged -9.25 to 10.25). For RAI, the Score 0 represents the crossover between more controlled motivation and more autonomous motivation, -9 the lower threshold, and 9 the upper threshold. Table 2 shows the frequency of cases after the configurations. The majority of children $(89 \%, 48 / 9)$ experienced high levels of SI in PE and 70\% (46/2) experienced $\mathrm{PE}$ as pedagogically inclusive (PI). More children were physically included (PHY) than excluded in PE, and a large proportion of the children experienced the PE climate to be mastery oriented (MAS) and autonomy supportive (AUS). Furthermore, the majority of children were highly task oriented (TAS) and experienced satisfaction of need for relatedness (REL) and autonomy (AUT) in PE, although 39\% (2/19) did not experience satisfaction of the need for competence.

\section{First Step: Analyses of Relationships Between Motivational Climates and Inclusion}

Truth tables for the two outcomes were constructed by grouping cases with similar configurations of the contextual conditions and indicating the outcome (OUT) associated with each configuration. The aim of the truth table is to examine cases that share similar conditions to see if they also share the same outcome (Ragin, 2008). To increase the robustness of the analyses, the minimum frequency of cases within a configuration was set to two, thus, excluding all configurations covering only one case. Table 3 shows five configurations with consistency above 0.85 for 
Table 2 Frequency of Cases by Membership Score

\begin{tabular}{|c|c|c|c|c|c|c|}
\hline Condition & $\leq \mathrm{LT}$ & $>\mathrm{LT}<\mathrm{CT}$ & $=\mathrm{CT}$ & $<\mathrm{UT}>\mathrm{CT}$ & $\geq \mathbf{U T}$ & Distinct value \\
\hline \multicolumn{7}{|l|}{ Outcome } \\
\hline SI & 1 & 3 & 3 & 48 & 9 & 19 \\
\hline PI & 1 & 10 & 5 & 46 & 2 & 22 \\
\hline \multicolumn{7}{|l|}{ Contextual } \\
\hline MAS & 3 & 11 & 4 & 29 & 17 & 12 \\
\hline PER & 13 & 18 & 6 & 21 & 6 & 13 \\
\hline AUS & 1 & 14 & 5 & 42 & 2 & 21 \\
\hline PHY & 2 & 1 & 1 & 11 & 49 & 5 \\
\hline \multicolumn{7}{|l|}{ Individual } \\
\hline TAS & 1 & 0 & 1 & 29 & 33 & 8 \\
\hline EGO & 3 & 19 & 4 & 20 & 18 & 13 \\
\hline AUT & 1 & 10 & 1 & 48 & 4 & 16 \\
\hline $\mathrm{COM}$ & 2 & 19 & 4 & 36 & 3 & 20 \\
\hline REL & 1 & 7 & 2 & 33 & 21 & 15 \\
\hline RAI & 1 & 14 & 1 & 45 & 3 & 40 \\
\hline AMO & 28 & 24 & 2 & 6 & 4 & 14 \\
\hline
\end{tabular}

Note . $\mathrm{LT}=$ lower threshold, $\mathrm{CT}=$ crossover threshold; $\mathrm{UT}=$ upper threshold; $\mathrm{SI}=$ social inclusion; $\mathrm{PI}=$ pedagogical inclusion; $\mathrm{MAS}=$ mastery climate $\mathrm{PER}=$ performance climate $; \mathrm{AUS}=$ autonomy support; $\mathrm{PHY}=$ physical inclusion; $\mathrm{TAS}=$ task orientation; $\mathrm{EGO}=$ ego orientation; $\mathrm{AUT}=$ need for autonomy; $\mathrm{COM}$ = need for competence; $\mathrm{REL}=$ need for relatedness; $\mathrm{RAI}=$ relative autonomy index; $\mathrm{AMO}=$ amotivation.

Table 3 Truth Tables of the Contextual Conditions for Inclusion in PE

\begin{tabular}{|c|c|c|c|c|c|c|c|c|c|c|}
\hline \multirow[b]{2}{*}{$n$} & \multirow[b]{2}{*}{ MAS } & \multirow[b]{2}{*}{ PER } & \multirow[b]{2}{*}{ AUS } & \multirow[b]{2}{*}{ PHY } & \multicolumn{3}{|c|}{ Social inclusion } & \multicolumn{3}{|c|}{ Pedagogical inclusion } \\
\hline & & & & & OUT & Con. & PRI & OUT & Con. & PRI \\
\hline 18 & 1 & 1 & 1 & 1 & 1 & 0.996 & 0.995 & 1 & 0.990 & 0.983 \\
\hline 11 & 1 & 0 & 1 & 1 & 1 & 0.987 & 0.980 & 1 & 0.979 & 0.954 \\
\hline 7 & 0 & 0 & 1 & 1 & 1 & 0.965 & 0.937 & 1 & 0.963 & 0.885 \\
\hline 6 & 1 & 1 & 0 & 1 & 1 & 0.993 & 0.987 & 1 & 0.955 & 0.840 \\
\hline 6 & 1 & 0 & 0 & 1 & 1 & 0.973 & 0.949 & 1 & 0.960 & 0.864 \\
\hline
\end{tabular}

Note. . Consistency cutoff $=0.85$, number of cases cutoff $=2, N=48$. MAS $=$ mastery climate; $\mathrm{PER}=$ performance climate; AUS = autonomy support; $\mathrm{PHY}=$ physical inclusion; OUT = outcome; Con. = consistency; PRI = proportional reduction in consistency.

both SI and PI (i.e., a minimum of $85 \%$ of the cases with the outcome also exhibit the conditions). All configurations display high consistency $(>0.97)$. The first two configurations represent the majority of the children in the analyses. The first configuration represents children who are physically included (PHY) in PE and 
who perceive the climate to be both performance (PER) and mastery oriented (MAS), as well as autonomy supportive (AUS) $(n=18)$. The next configuration represents physically included children who perceived the climate to be mastery oriented and autonomy supportive, but low on performance orientation $(n=11)$.

The analyses of necessity relations, depicted in Table 4, identified the contextual conditions present whenever children perceive the PE climate as inclusive. ${ }^{1}$ Several of the necessary configurations are combined with the logical OR (+) operator, also referred to as union in which at least one of the conditions needs to be present for children to feel included in PE. As Table 4 shows physical inclusion was the only single contextual condition that met the criteria of necessity for both SI and PI in PE. This supports our assumption that perceived PI and SI necessitates that children are more physically included than excluded.

For SI, the following four configurations indicated that both a masteryoriented and an autonomy-supportive climate promote SI in union with each other or with a low performance-oriented climate. Necessary relations between contextual conditions and PI yielded four additional unions, in which two unions included the presence of a performance climate; and two unions included low mastery climate or low physical inclusion. ${ }^{2}$ Identification of necessity relations does not rule out that a necessary configuration can be present without the child

\section{Table 4 Necessity Relations Between Motivational Climates and Inclusion in Physical Education}

\begin{tabular}{lccccc}
\hline & Con. & Cov.r & Negations & Con. & Cov.r \\
\hline SI & & & & & \\
PHY & 0.935 & 0.872 & Phy & 0.124 & 0.732 \\
MAS+per & 0.935 & 0.868 & mas+PER & 0.160 & 0.971 \\
MAS+aus & 0.858 & 0.901 & mas+AUS & 0.265 & 0.916 \\
MAS+AUS & 0.903 & 0.914 & mas+aus & 0.222 & 0.873 \\
per+AUS & 0.870 & 0.886 & PER+aus & 0.259 & 0.994 \\
PI & & & & & \\
PHY & 0.952 & 0.772 & Phy & 0.144 & 0.739 \\
MAS+per & 0.961 & 0.776 & mas+PER & 0.182 & 0.959 \\
MAS+PER & 0.865 & 0.838 & mas+per & 0.322 & 0.812 \\
mas+AUS & 0.866 & 0.876 & MAS+aus & 0.407 & 0.923 \\
MAS+aus & 0.903 & 0.824 & mas+AUS & 0.305 & 0.917 \\
MAS+AUS & 0.948 & 0.834 & mas+aus & 0.259 & 0.882 \\
MAS+phy & 0.885 & 0.816 & mas+phy & 0.309 & 0.898 \\
per+AUS & 0.932 & 0.825 & PER+aus & 0.285 & 0.951 \\
PER+AUS & 0.882 & 0.898 & per+aus & 0.401 & 0.896 \\
\hline
\end{tabular}

Note. . Consistency cutoff $=0.85$, coverage cutoff $=0.6$, number of cases cutoff $=2$. SI $=$ social inclusion; $\mathrm{PI}=$ pedagogical inclusion; $\mathrm{MAS}=$ mastery climate; $\mathrm{PER}=$ performance climate; AUS = autonomy support; $\mathrm{PHY}=$ physical inclusion; $\mathrm{PRI}=$ proportional reduction in consistency; con. = consistency; cov.r = raw coverage. 
feeling included. To identify sufficient conditions for children to feel included, we need to explore sufficiency relations.

With the use of Boolean minimization, we reduced the complexity of the truth tables (Table 3) to produce a formula for minimal sufficient configurations of the outcomes. The minimization of the contextual conditions indicated a model consisting of two sufficient configurations for both SI and PI:

$$
\begin{gathered}
\mathrm{SI} \leftarrow \mathrm{MAS} * \mathrm{PHY}+\text { per } * \mathrm{AUS} * \mathrm{PHY} \\
\mathrm{PI} \leftarrow \mathrm{MAS} * \mathrm{PHY}+\operatorname{per} * \mathrm{AUS} * \mathrm{PHY}
\end{gathered}
$$

For children with disabilities to feel socially and pedagogical included in PE, they need to be either physically included and experience the climate as mastery oriented (cov.u $=0.378$ in the SI model, cov.u $=0.367$ in the PI model), or physically included and experiencing the climate as autonomy supportive and not performance oriented (cov.u $=0.070$ in the SI model, cov.u $=0.075$ in the PI model). Furthermore, the models indicate physical inclusion as necessary, but not sufficient condition for inclusion. The difference between SI- and PI-supportive conditions were in the model fit-with the overall fit of the model for SI (incl= $0.938, \mathrm{PRI}=0.923$, cov.r $=0.848$ ) performing slightly better than the model for PI in terms of consistency (incl $=0.864$, PRI $=0.799$, cov.r $=0.898$ ). As all configurations were theoretically sound and well supported by the empirical data, the inclusion-supportive conditions were included in the second step of the analyses.

\section{Second Step: Relationship Between Inclusion-Supportive Climates and Individual Attributes}

In the second step, we explored which combinations of individual attributes (i.e., task orientation, ego orientation, the satisfaction of autonomy, competence, and relatedness, RAI, and amotivation) within the inclusion-supportive climates that jointly supported perceptions of SI and PI in PE. The results after the Boolean minimization are shown in Table 5. Table 5 illustrates the sufficient paths toward inclusion in PE. Each path indicates the combination of contextual conditions and individual attributes supportive of SI and PI among children with disabilities. Table 5 exemplifies several strengths of the QCA approach. First, fsQCA allowed us to identify various pathways to inclusion in PE. The results indicated four inclusion-supportive paths for both SI and PI that differed slightly in model fit for the two outcomes. For example, take the paths S3/P3 with the largest coverage of children within the MAS*PHY climate, these paths comprise the group of children who were task and ego oriented, low on amotivation, and experienced satisfaction of the need for autonomy, competence, and relatedness

Second, fsQCA allowed us to explore whether conditions on their own or in combinations were sufficiently associated with inclusion in PE. Table 5 indicates that all paths consisted of the intersection of six or more attributes. In line with a multifaceted understanding of inclusion in PE, no single condition was sufficient to explain satisfaction with SI or PI on their own, only in combination with other conditions. 


\section{Table 5 Sufficient Paths Toward Social and Pedagogical Inclusion in Physical Education}

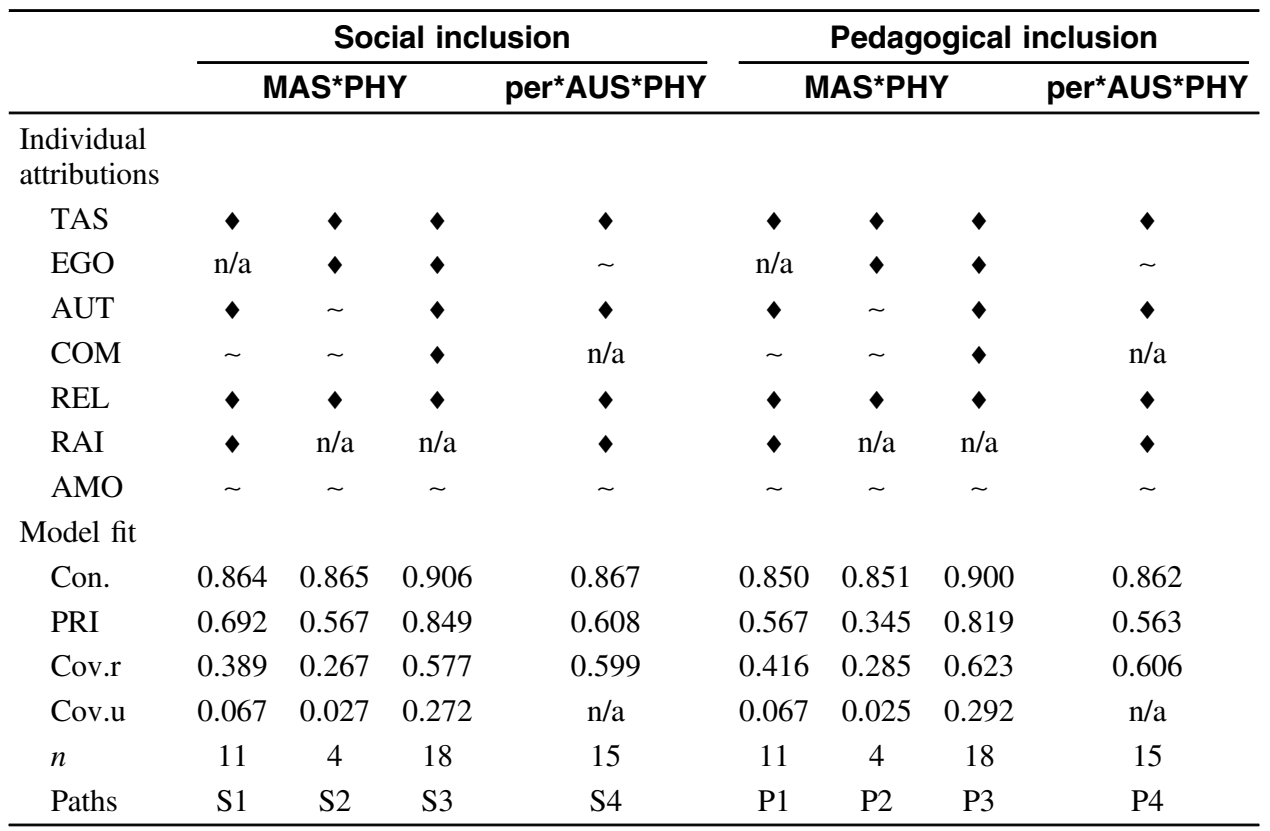

Note. $\quad$ = membership in the condition; $\sim=$ nonmembership in the condition; $\mathrm{n} / \mathrm{a}=$ not applicable/not sufficient condition for the identified configuration. $\mathrm{SI} * \mathrm{MAS} * \mathrm{PHY}$ paths: con. cut $=0.85$, con $=0.894, \mathrm{PRI}=0.830$, cov $\cdot \mathrm{r}=$ $0.699, n=37$, multiple covered cases $=0$. SI*per*AUS*PHY paths: con. cut $=0.85$, con. $=0.867, \mathrm{PRI}=0.608$, cov.r $=0.599, n=37$, multiple covered cases $=0$. PI*MAS*PHY paths: con.cut $=0.85$, con. $=0.876, \mathrm{PRI}=$ 0.772 , cov.r $=0.744, n=37$, multiple covered cases $=0$. PI*per*AUS*PHY paths: con.cut $=085$, con. $=$ $0.862, \mathrm{PRI}=0.563$, cov.r $=0.606, n=37$, multiple covered cases $=0$. MAS $=$ mastery climate; AUS = autonomy support; $\mathrm{PHY}=$ physical inclusion; TAS = task orientation; EGO = ego orientation; AUT = need for autonomy; $\mathrm{COM}=$ need for competence; $\mathrm{REL}=$ need for relatedness; $\mathrm{RAI}=$ relative autonomy index; $\mathrm{AMO}=$ amotivation; $\mathrm{PRI}=$ proportional reduction in consistency; $\operatorname{cov} . \mathrm{u}=$ unique coverage ; cov.r $=$ raw coverage; con. cut $=$ consistency cutoff; con = consistency.

Third, both the presence and absence of a condition supported inclusion in PE dependent on the different motivational pathways. An example is how the presence (see paths S1/P1, S3/P3, and S4/P4) and absence (see S2/P2) of the need satisfaction of autonomy (AUT) was a supportive condition for inclusion in PE depending on the path.

If we look at the paths combined, we see that three attributes promoted SI and PI in all configurations, namely task orientation, satisfaction of relatedness, and low levels of amotivation. All other variables were INUS conditions (i.e., insufficient but necessary part of a condition which itself is unnecessary but sufficient for the result). For example, take the need for competence satisfaction $(\mathrm{COM})$ in path S3. First, S3 children's satisfaction of competence was an insufficient attribute to explain SI in itself (TAS, EGO, AUT, REL, and amo were also required), yet it was a necessary condition within S3. Second, S3 was a 
sufficient, but not necessary, path given that presence of the paths S2 and S3 that also supported SI. The INUS conditions exemplify that inclusion in PE is seldom a result of independent conditions-which is a common assumption in traditional statistical inference-rather the significance of a condition often depends on the co-occurrence of other conditions.

The paths S1-S3 and P1-P3 within the physically inclusive and masteryoriented climate indicated that children with high or low competence need satisfaction experienced SI and PI in PE dependent on the configuration with other attributes. S1/P1 indicated that children experiencing low competence satisfaction experienced SI and PI if they were task oriented, autonomously motivated, low on amotivation, and experienced the needs satisfaction of autonomy and relatedness. Ego orientation was an irrelevant attribute in these paths, which indicate that children high and low on ego orientation could experience inclusion as long as the other attributes were present. S2/P2 indicated that the children with disabilities who did not experience satisfaction of competence and autonomy still experienced being socially and pedagogically included if they were task and ego oriented, low on amotivation, and the need for relatedness was secured. The third paths in the physical inclusive and mastery-oriented climate, S3/P3 represent the children that were task and ego oriented, low on amotivation, and experienced satisfaction of all three basic needs. Moreover, children's motivational regulation was irrelevant for the children with the motivational profiles described in S2/P2, and S3/P3.

The second inclusion-supportive context comprised of the physically inclusive, autonomy-supportive, and low-performance orientation climate yielded one path for both necessary and sufficient for SI and PI (S4/P4). Within this climate, children experienced SI and PI if they were task oriented, not ego oriented, intrinsically motivated, low on amotivation, and experienced satisfaction of the need for autonomy and relatedness.

\section{Sensitivity Analyses and Robustness of the Results}

Qualitative comparative analysis have recently been critiqued for parameter sensitivity (Skaaning, 2011). To test for parameter sensitivity, we performed several sensitivity analyses of the models by changing (a) the crossover threshold of calibration of the outcomes $(2.9,3.1)$; (b) the consistency cutoff $(0.8,0.90)$ in the analyses; and (c) the frequency of cases $(1,3)$ linked to the configurations (Skaaning, 2011). The tests indicated that the contextual analyses were robust, but the final models introducing the individual attributes were more sensitive to changes in consistency and frequency thresholds. One solution could be to increase the consistency threshold to 0.90 . This would result in two paths instead of four for both SI and PI, with the paths being more robust to change in frequency thresholds. However, consistency threshold of 0.9 is not compelling because it would decrease the coverage score of the models significantly (Ragin, 2008). Furthermore, allowing paths with one case decreases the robustness of the solutions in most models. As a result, we kept the cutoff of minimum two cases for the configurations and the consistency threshold of 0.85 . This is well within the range of consistency scores often used in fsQCA. ${ }^{3}$ 


\section{Discussion}

The two-step fsQCA analyses indicated two sufficient inclusion-supportive contextual conditions and four sufficient motivational paths toward SI and PI.

\section{Contextual Conditions That Foster SI and PI in PE}

This study indicates that for children to feel socially and pedagogically included, it is necessary for them to be more physically included than excluded from the PE lessons, but physical inclusion is not sufficient by itself. For SI and PI to be achieved, children also need to experience the climate to be either mastery oriented or autonomy supportive and low on performance orientation. These findings are in line with both AGT and SDT and complement previous research that found both autonomy supportive and mastery-oriented climates to positively influence children's experience of enjoyment and low levels of amotivation (Ommundsen \& Kvalø, 2007; Spray et al., 2006), and aid learning for children with and without disabilities (Valentini \& Rudisill, 2004).

A new finding is that an autonomy-supportive environment does not promote SI and PI alone but does so in combination with a physically inclusive and low performance-oriented climate. Normative conceptions of ability and valuation of an ideal body and motor competence are inevitable in performance-oriented competitive environments (Fitzgerald, 2005; Nicholls, 1979). These contextual conditions do not promote appreciation of diversity or mastery experiences within a heterogeneous group of children. Thus, for children to feel socially and pedagogically included, it seems particularly important to facilitate a masteryoriented climate or to reduce the performance-oriented criteria in PE.

In addition, common approaches to special education and adapted PE are least restrictive environment approaches and segregated teaching with an overall aim of inclusion in the future. Such efforts might not be without value, but, as Nicholls (1979) reminded us, they do not alter the PE context or the contributing factors of inequality in motivation and education. If we understand disability as the outcome of the interaction between individual attributes and contextual conditions, as in the interactional approach to disability (Shakespeare, 2006), it becomes important to explore how we can alter the way PE is taught in response to the needs and abilities represented within the group of children, rather than to exclude children who do not "fit within" normative conceptions of ability (Fitzgerald, 2005).

\section{SI- and PI-Supportive Paths in PE}

The inclusive-supportive context physically inclusive, autonomy-supportive, and low performance-oriented climate yielded one path that sufficiently explained SI and PI. While the inclusion-supportive context physical inclusive and masteryoriented climate appears more robust, in that it yielded three different motivational pathways, which allows children with different motivational profiles to feel included in PE.

In line with previous research, we found that the combination of task orientation, low amotivation, and relatedness need satisfaction were adaptive motivational attributes in PE (Cox \& Williams, 2008; Standage et al., 2003). 
The different paths indicate that children with different levels of satisfaction of the needs for competence and autonomy could feel both socially and pedagogically included as long as they were task oriented, low on amotivation, and experiences satisfaction of the need for relatedness. This was the case for children both high and low on ego orientation, and in some paths, these relations were also independent of the level of autonomous motivation. In other words, satisfaction of all three basic psychological needs was not necessary for children to feel included in PE. This is essential when we consider that a large proportion of the children in this study did not experience the fulfilment of the need for competence or autonomy in PE. One interpretation is that a mastery climate or absence of a performance climate reduces the importance of the need for competence satisfaction because effort and learning are in focus instead of normative ability.

The different paths also enrich our understanding of the orthogonal nature of ego and task orientation. In the paths identified in Table 5, we see that childrenboth ego and task oriented or only task oriented-experienced SI and PI within a physically inclusive and mastery-oriented climate. This supports studies that emphasized high task and high ego orientation, or high task and low ego orientation as adaptive motivational attributes (Roberts, 2012).

In terms of practical implication, the TARGET approach (task, authority, recognition, grouping, evaluation, and time) attributed to Epstein (1987) and recommended by Ames (1992), can provide guidance for modifying learning climates that accommodate diversity in PE (Valentini \& Rudisill, 2004). To promote feelings of inclusion, the tasks should be mastery-oriented, give sufficient time for children to learn and execute the tasks, and allow for task novelty and variation. Effort and progress should be the salient ingredients in recognitions and evaluations (Ames, 1992; Roberts, 2012). In terms of the grouping and the importance of satisfaction of relatedness found in this study, children with disabilities may benefit from more opportunities to collaborate, and form positive peer relationships in PE. Finally, in line with the authority structure to provide children with optimal challenging activities, the PE teacher should provide choices guided by children's interests and abilities in a structured environment (Ames, 1992; Deci \& Ryan, 2000).

\section{Limitations of the Study}

Several limitations apply to this study. The analyses are based on convenience sampled cross-sectional data, which limits the possibility for causal inference and to generalization. It would be interesting to follow a group of children with disabilities, and their peers and teachers, longitudinally to further explore the relations between alterations in the PE settings and the children's degree of participation, the motivational processes, and its influence of children's feelings of inclusion. Furthermore, in line with the asymmetrical assumptions of QCA, this study explores the necessary and/or sufficient conditions for children to feel socially and pedagogically included and does not make assumptions about factors that might lead to social and pedagogically exclusion or marginalization. Please note that the children in this study were enrolled in general schools, and the majority of the children attended PE together with their peers to some degree. Thus, this study is limited to this population and does not say anything about the children who attend segregated PE programs or who are completely excluded from PE. 


\section{Theoretical Implications}

fsQCA supported the identification of previously unexplored complex relations between the different tenets that might complement contemporary theoretical discussions. First, the second contextual inclusion-supportive climate indicated for children to feel socially and pedagogically included in PE is not enough that the climate is autonomy supportive-it also needs to be low on performance orientation. Thus, the achievement goal climate, and subsequently the applied criteria of success, seems to be of particular importance for creating inclusion-supportive climates in PE.

Second, SDT postulates that the degree of basic psychological needs satisfaction, especially the need for competence and autonomy, dictates the level of selfdetermined behavior (Deci \& Ryan, 2000; Standage et al., 2003). With this in mind, we could expect that children with high level of basic needs satisfaction would exhibit high level of autonomous motivation. However, one path (S3/P3) to both SI and PI indicated that the level of autonomous motivation was not applicable in the configuration where children experienced satisfaction of all three psychological needs. In other words, children with both controlled and autonomous motivations experienced satisfaction of the need for autonomy, relatedness, and competence dependent on the motivational climate and the presence of other individual attributes such as their motivational orientation and level of amotivation. This does not invalidate the satisfaction of the three basic needs as a possible sufficient pathway for autonomous motivation, but it suggests that there may be alternative pathways to autonomous motivation that do not depend on the satisfaction of all three psychological needs. To explore the complex asymmetrical and equifinality of motivational processes further, we recommend that several studies retain a QCA approach, alone or in combination with more traditional statistical analyses.

\section{Conclusion}

The findings contribute to our understanding of how PE teachers can foster motivational climates that promote SI and PI for a diverse group of children. First, it is necessary for children to be physically included to feel socially and pedagogically included in PE. Second, the introduction of a mastery-oriented climate and/or an autonomy-supportive climate, low on performance orientation seems to be successful motivational strategies for SI and PI in PE. A mastery climate seems to be a particular robust inclusion-supportive climate for children with different motivational profiles and abilities. The findings illustrate the practical and theoretical value of applying fsQCA to explore motivational pathways in PE using tenets from AGT and SDT.

\section{Notes}

1. Necessary conditions are identified if the individual's membership score in the condition is equal to or higher than the individual's score on the outcome.

2. We tested for paradoxical relations in which the presence of a condition/configuration, as well as its negation (its absence), explain the outcome. No paradoxical relations emerged based on consistency scores far below the threshold (0.85).

3. Results of the sensitivity and robustness tests are available upon request. 


\section{References}

Ames, C. (1992). Classrooms: Goals, structures, and student motivation. Journal of Educational Psychology, 84(3), 261-271. doi:10.1037/0022-0663.84.3.261

Booth, T., \& Ainscow, M. (2002). Index for inclusion: Developing learning and participation in schools. Bristol, England: Centre for Studies on Inclusion Education.

Braithwaite, R., Spray, C.M., \& Warburton, V.E. (2011). Motivational climate interventions in physical education: A meta-analysis. Psychology of Sport and Exercise, 12(6), 628-638. doi:10.1016/j.psychsport.2011.06.005

Cooper, B., \& Glaesser, J. (2011). Using case-based approaches to analyse large datasets: A comparison of Ragin's fsQCA and fuzzy cluster analysis. International Journal of Social Research Methodology, 14(1), 31-48. doi:10.1080/13645579.2010. 483079

Cox, A., \& Williams, L. (2008). The roles of perceived teacher support, motivational climate, and psychological need satisfaction in students' physical education motivation. Journal of Sport \& Exercise Psychology, 30(2), 222-239. PubMed ID: 18490792 doi:10.1123/jsep.30.2.222

Dalen, M. (1994). Så langt det er mulig og forsvarlig: Integrering av funksjonshemmede I grunnskolen. [As far as it is possible and professionably advisable: Integration of individuals with disabilities in school]. Oslo, Norway: Universitetsforlaget.

Deci, E.L., \& Ryan, R.M. (2000). The "what" and "why" of goal pursuits: Human needs and the self-determination of behavior. Psychological Inquiry, 11(4), 227-268. doi:10. 1207/S15327965PLI1104_01

Duda, J.L. (2013). The conceptual and empirical foundation of Empowering Coaching ${ }^{\mathrm{TM}}$ : Setting the stage for the PAPA project. International Journal of Sport and Exercise Psychology, 11(4), 311-318. doi:10.1080/1612197X.2013.839414

Dunn, J.C., \& Dunn, J.G.H. (2006). Psychosocial determinants of physical education behavior in children with movement difficulties. Adapted Physical Activity Quarterly, 23, 293-309. doi:10.1123/apaq.23.3.293

Dusa, A. (2007). User manual for the QCA(GUI) package in R. Journal of Business Research, 60(5), 576-586. doi:10.1016/j.jbusres.2007.01.002

Edmunds, J.K., Ntoumanis, N., \& Duda, J.L. (2007). Perceived autonomy supportive and psychological need satisfaction in exercise. In M.S. Hagger \& N.L.D. Chatzisarantis (Eds.), Intrinsic motivation and self-determination in exercise and sport. Champaign, IL: Human Kinetics.

Epstein, J.L. (1987). TARGET: An examination of parallel school and family structures that promote student motivation and achievement (Report No. 6). Baltimore, MD: Johns Hopkins University.

Fitzgerald, H. (2005). Still feeling like a spare piece of luggage? Embodied experiences of (dis)ability in physical education and school sport. Physical Education and Sport Pedagogy, 10(1), 41-59. doi:10.1080/1740898042000334908

Goudas, M., Biddle, S.J.H., \& Fox, K. (1994). Perceived locus of causality, goal orientations, and perceived competence in school physical education classes. British Journal of Educational Psychology, 64, 453-463. PubMed ID: 7811633 doi:10.1111/j.20448279.1994.tb01116.x

Grolnick, W.S., \& Ryan, R.M. (1987). Autonomy in children's learning: An experimental and individual difference investigation. Journal of Personality and Social Psychology, 52, 890-898. PubMed ID: 3585701 doi:10.1037/0022-3514.52.5.890

Hastie, R., Rudisill, E.M., \& Wadsworth, D.D. (2013). Providing students with voice and choice: Lessons from intervention research on autonomy-supportive climates in physical education. Sport, Education and Society, 18(1), 38-56. doi:10.1080/13573322. 2012.701203 
Hein, V., \& Koka, A. (2007). Perceived feedback and motivation in physical education and physical activity. In M.S. Hagger \& N.L.D. Chatzisarantis (Eds.), Intrinsic motivation and self-determination in exercise and sport (pp. 127-140). Champaign, IL: Human Kinetics.

Jaakkola, T., Wang, C.K.J., Soini, M., \& Liukkonen, J. (2015). Students' perceptions of motivational climate and enjoyment in Finnish physical education: A latent profile analysis. Journal of Sports Sciences \& Medicine, 14(3), 477-483.

Klavina, A., \& Block, M.E. (2008). The effect of peer tutoring on interaction behaviors in inclusive physical education. Adapted Physical Activity Quarterly, 25, 132-158. doi:10.1123/apaq.25.2.132

Mageau, G.A., \& Vallerand, R.J. (2003). The coach-athlete relationship: A motivational model. Journal of Sports Sciences, 21, 883-904. doi:10.1080/0264041031000140374

Nicholls, J.G. (1979). Quality and equality in intellectual development: The role of motivation in education. American Psychologist, 34(11), 1071-1084. doi:10.1037/ 0003-066X.34.11.1071

Nicholls, J.G. (1989). The competitive ethos and democratic education. Cambridge, UK: Harvard University Press.

Ntoumanis, N., \& Biddle, S.J.H. (1999). Affect and achievement goals in physical activity: A meta-analysis. Scandinavian Journal of Medicine \& Science in Sports, 9(6), 315-332. doi:10.1111/j.1600-0838.1999.tb00253.x

Obrusnikova, I., \& Dillon, S.R. (2012). Students' beliefs and intentions to play with peers with disabilities in physical education: Relationships with achievement and social goals. Journal of Teaching in Physical Education, 31, 311-328. doi:10.1123/jtpe.31.4.311

Ommundsen, Y., \& Kvalø, S.E. (2007). Autonomy-mastery, supportive or performance focused. Different teacher behaviours and pupils' outcome in physical education. Scandinavian Journal of Educational Research, 51(4), 385-413. doi:10.1080/ 00313830701485551

Pan, C.Y., Tsai, C.L., Chu, C.H., \& Hsieh, K.W. (2011). Physical activity and selfdetermined motivation of adolescents with and without autism spectrum disorders in inclusive physical education. Research in Autism Spectrum Disorders, 5, 733-741. doi:10.1016/j.rasd.2010.08.007

Parish, L.E., \& Treasure, D.C. (2003). Physical activity and situational motivation in physical education: Influence of the motivational climate and perceived ability. Research Quarterly for Exercise and Sport, 74(2), 173-182. PubMed ID: 12848230 doi:10. 1080/02701367.2003.10609079

Ragin, C.C. (2008). Redesigning social inquiry: Fuzzy sets and beyond. Chicago, IL: University of Chicago Press.

Roberts, G.C. (2012). Motivation in sport and exercise from an achievement goal theory perspective: After 30 years, where are we? In G.C. Robert \& D.C. Treasure (Eds.), Advances in motivation in sport and exercise (3rd ed., pp. 5-58). Champaign, IL: Human Kinetics.

Ryan, R.M., \& Deci, E.L. (2002). Overview of self-determination theory: An organismic dialectical perspective. In E.L. Deci \& R.M. Ryan (Eds.), Handbook of self-determination research (pp. 3-36). Rochester, NY: University of Rochester Press.

Schneider, C.Q., \& Wagemann, C. (2006). Reducing complexity in qualitative comparative analysis (QCA): Remote and proximate factors and the consolidation of democracy. European Journal of Political Research, 45(5), 751-786. doi:10.1111/j.1475-6765. 2006.00635.x

Seymour, H., Reid, G., \& Bloom, G.A. (2009). Friendship in inclusive physical education. Adapted Physical Activity Quarterly, 26, 201-219. PubMed ID: 19799094 doi:10. 1123/apaq.26.3.201

Shakespeare, T. (2006). Disability rights and wrongs. Abingdon, England: Routledge. 
Skaaning, S.E. (2011). Assessing the robustness of crisp-set and fuzzy-set QCA results. Social Methods \& Research, 40(2), 391-408. doi:10.1177/0049124111404818

Sørensen, M., Roberts, G.C., \& Farholm, A. (2018). Motivational climate in the home: Implications for physical activity, well-being and family relations. Manuscript submitted for publication.

Spencer-Cavaliere, N., \& Watkinson, E.J. (2010). Inclusion understood from the perspectives of children with disability. Adapted Physical Activity Quarterly, 27, 275-293. PubMed ID: 20956835 doi:10.1123/apaq.27.4.275

Spray, C.M., Wang, J.C.K., Biddle, S.J.H., \& Chatzisarantis, N.L.D. (2006). Understanding motivation in sport: An experimental test of achievement goal and self-determination theories. European Journal of Sport Science, 6(1), 43-51. doi:10.1080/17461390 500422879

Standage, M., Duda, J.L., \& Ntoumanis, N. (2003). A model of contextual motivation in physical education: Using constructs from self-determination and achievement goal theories to predict physical activity intentions. Journal of Educational Psychology, 95(1), 97-110. doi:10.1037/0022-0663.95.1.97

Standage, M., Duda, J.L., \& Ntoumanis, N. (2006). Students' motivational processes and their relationship to teacher ratings in school physical education: A self-determination theory approach. Research Quarterly for Exercise and Sport, 77(1), 100-110. doi: 10.1080/02701367.2006.10599336

Tabachnick, B.G., \& Fidell, L.S. (2014). Using multivariate statistics. Essex, England: Pearson.

Thiem, A., \& Dusa, A. (2013). Qualitative comparative analysis with R: A user's guide. New York, NY: Springer.

Valentini, N.C., \& Rudisill, M.E. (2004). An inclusive mastery climate intervention and the motor skill development of children with and without disabilities. Adapted Physical Activity Quarterly, 21, 330-347. doi:10.1123/apaq.21.4.330

Van Buuren, S., \& Groothuis-Oudshoorn, K. (2011). MICE: Multivariate imputation by chained equations in R. Journal of Statistical Software, 45(3), 1-67. doi:10.18637/jss. v045.i03

Van den Berghe, L., Vansteenkiste, M., Cardon, G., Kirk, D., \& Haerens, L. (2014). Research on self-determination in physical education: Key findings and proposals for future research. Physical Education and Sport Pedagogy, 19(1), 97-121. doi:10.1080/ 17408989.2012.732563

Wilhelmsen, T., \& Sørensen, M. (2017). Inclusion of children with disabilities in physical education: A review of literature from 2009 to 2015. Adapted Physical Activity Quarterly, 34, 311-337. doi:10.1123/apaq.2016-0017 